\title{
Energy-Efficient WDM Network Planning with Dedicated Protection Resources in Sleep Mode
}

\author{
Ajmal Muhammad ${ }^{1}$, Paolo Monti ${ }^{2}$, Isabella Cerutti ${ }^{3}$, Lena Wosinska ${ }^{2}$, Piero Castoldi $^{3}$, Anna Tzanakaki $^{4}$ \\ ${ }^{1}$ Linköping University, Linköping, Sweden, Email: ajmal@isy.liu.se \\ ${ }^{2}$ Royal Institute of Technology (KTH), Stockholm, Sweden, Email: \{pmonti,wosinska\}@kth.se \\ ${ }^{3}$ Scuola Superiore Sant'Anna, Pisa, Italy, Email: \{i.cerutti,p.castoldi\}@ sssup.it \\ ${ }^{4}$ Athens Information Technology (AIT), Peania, Greece, Email: atza@ait.edu.gr
}

\begin{abstract}
Energy-efficient optical networks are gaining momentum as environmental-friendly solutions with reduced operational costs. Energy-efficiency can be achieved by using devices in sleep mode, i.e., a low-power, inactive state in which devices can be suddenly waken-up upon occurrence of triggering events.

This paper advocates a sleep mode option for the optical devices (e.g., amplifiers, optical switches) installed for protection purposes only. These devices can be put in sleep mode to reduce the network power consumption, but they can be promptly waken up (if necessary) upon a failure occurrence. This principle is proposed and applied in Wavelength Division Multiplexing (WDM) networks with dedicated-path protection to ensure survivability against single-link failures.

The main contribution of the paper is the definition of the energy-efficient network planning problem for resilient WDM networks where optical devices can be configured in sleep mode. Optimal results of the integer linear programming (ILP) problem show savings of up to $25 \%$ in the overall power consumption.
\end{abstract}

\section{INTRODUCTION}

Due to the continuous increase of traffic, power consumption is becoming a significant contribution to the operational expenditure of telecommunications networks with a nonnegligible contribution to the global warming effects [1]. In optical WDM networks, an energy-efficient planning of the resources to be provisiond for supporting the requested optical connection, or lightpaths, can reduce considerably the overall power consumption [2]-[5].

In addition to provisioning resources for the lightpaths supporting traffic (i.e., working lightpaths), additional redundant resources are required to overcome service interruptions due to unpredictable failures [6]. Such resources are typically maintained active, independently of the network status, and thus consume power even in the absence of failures. However, the currently available strategies for energy-efficient planning [2]-[5] lack to address the issue of provisioning redundant resources and to account for the corresponding power consumption.

In this paper, energy-efficient planning (i.e., static routing and wavelength assignment) of survivable WDM networks is considered for the first time. Energy-efficiency is achieved not

The work was supported by BONE ("Building the Future Optical Network in Europe"), a Network of Excellence funded by the European Commission through the 7th ICT-Framework Programme, and "All-optical overlay network" funded by VINNOVA (The Swedish Governmental Agency for Innovation Systems). only through a proper provisioning of the resources for working and protection lightpaths, but also through an innovative way of reducing the network power consumption of redundant resources. Since redundant resources are unused until a failure occurs, they can be set in sleep mode. Sleep mode represents a low-power, inactive state from which devices can be suddenly waken-up upon the occurrence of a triggering event. Although not available yet in most network devices, support of sleep mode is advocated by current efforts from standardization bodies and governmental programs [7].

In order to make sleep mode effective in survivable WDM networks, two issues need to be addressed. First, it is necessary to ensure that the devices in sleep mode can become promptly operative (typically within $50 \mathrm{~ms}$ ) in case of a failure. In this study, it is assumed that a mechanism for a prompt activation exists (e.g., through the development of suitable technology solutions and, if required, the use of the control plane). Second, it is necessary to ensure that working lightpaths are not supported by devices in sleep mode. Such issue requires a proper planning strategy.

The problem of planning a WDM network with dedicated 1:1 path protection [6] and devices supporting sleep mode is considered in this paper. For each connection request, a link-disjoint working and protection lightpaths need to be provisioned, in order to guarantee $100 \%$ single link failure survivability. The devices in links and at nodes can be put in sleep mode only if they are used exclusively for protection purposes. With this rationale, good candidates for sleep mode are devices installed in the links (e.g., in-line optical amplifiers) and at nodes, if traversed only by protection lightpaths.

The planning problem is formalized as an integer linear programming formulation, based on a general model for accounting the power consumption of the different operational modes of the devices. Such formulation is applied to an alloptical network with the aim to minimize the overall power consumption. Optimal solutions aim at evaluating the power saving that can be achieved by exploiting the sleep mode of the redundant devices allocated to support protection requirements through a suitable network planning approach.

\section{Sleep Mode of Path-Protection Resources}

Consider a WDM network in which optical devices operate in three different operational modes [7]: off, sleep, and active. 
Full (working) functionalities are available in active mode, while null, or close to null, functionalities are available in sleep and off modes. The difference between sleep mode and off mode is that the former permits a prompt transition to active mode.

\section{A. Power Model}

The amount of power consumed by the devices depends on their operational modes (Table I). When off, a device is disconnected from both the WDM network and the electrical network. When sleeping, a device consumes a negligible amount of power to ensure that it can be promptly switched to active mode at any moment. When active, a device drains an amount of power, dependent on its specific functionalities. It is assumed that devices in active mode are consuming a constant amount of power independent of their traffic load (fixed power), and an amount of power proportional to their traffic load (proportional power). Therefore, the total power consumption of an active device is the sum of the fixed power and the proportional power.

Table I

POWER CONSUMPTION OF THE OPERATIONAL MODES

\begin{tabular}{|c|l|l|}
\hline Mode & Functionality & Power Consumption \\
\hline \hline Off & Null & None \\
\hline Sleep & $\begin{array}{l}\text { Prompt switching to } \\
\text { active mode when triggered }\end{array}$ & Negligible \\
\hline Active & Full & Fixed power + proportional power \\
\hline
\end{tabular}

This generic power model can be applied to the optical devices installed in the network links (e.g., amplifiers) and the optical devices at the nodes (e.g., switches), where the proportional power is typically a function of the number of lightpaths. Notice that for some devices either the fixed power or the proportional power can be negligible (e.g., the proportional power in amplifiers).

The overall power drained by the WDM network is, thus, given by the power consumed by the active devices installed in links and nodes ${ }^{1}$. The impact of the operational modes on the overall power consumption of a WDM network with dedicated protection is discussed next.

\section{B. Protection Provisioning with Sleep Mode Option}

Consider a WDM network in which nodes are equipped with optical cross-connects (OXC) and optical wavelength converters available at each node. Resilience against single-link failure is provided through dedicated 1:1 path-protection. A set of (unidirectional) working lightpaths needs to be provisioned and protected by dedicated (link-disjoint) protection lightpaths.

An example of network planning is shown in Fig. 1, where four working lightpaths with their corresponding (linkdisjoint) protection lightpaths are routed. Devices in links $(L, G),(G, H),(G, F)$, and $(I, B)$ need to be active as they

\footnotetext{
${ }^{1}$ Although both sleeping and off devices are not accounted in the total network power consumption, their functionalities differ: sleeping devices can be quickly re-activated upon request, while off devices can not.
}

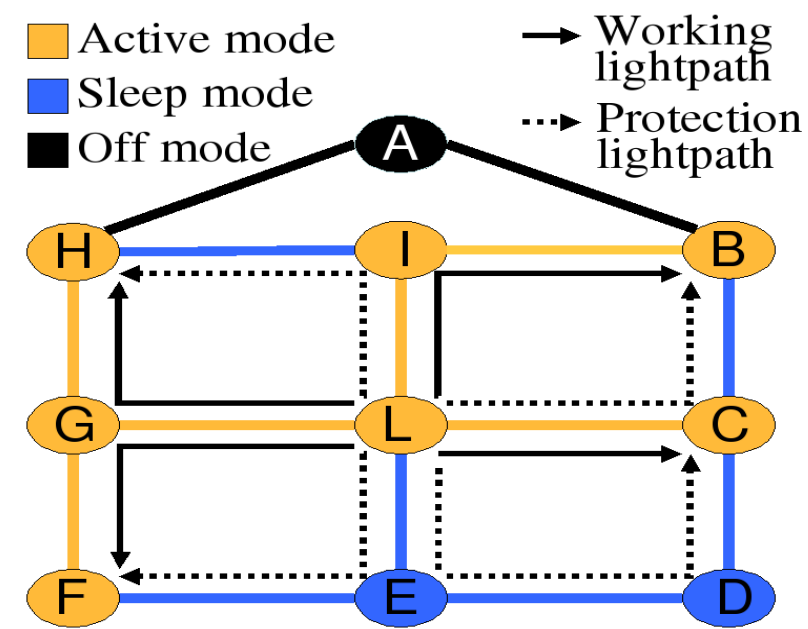

Figure 1. Impact of routing working and protection lightpaths and setting operational modes of devices.

support working lightpaths. Also, devices in links $(L, I)$ and $(L, C)$ need to be active as they support both working and protection lightpaths. To save power, devices in links $(A, H)$ and $(A, B)$ can be turned off. Also, devices in links $(L, E)$, $(E, F),(E, D),(D, C),(C, B)$, and $(I, H)$ are redundant as they need to ensure full working functionalities only in the case of failure. Therefore, they can be put in sleep mode.

Similar considerations can be derived for the devices at the nodes. Devices at node $A$ can be turned off, devices at nodes $D$ and $E$ can be in sleep mode, while devices at all other nodes must be in active mode.

Notice that by routing the working lightpath from node $L$ to node $B$ on links $(L, C)$ and $(C, B)$ (and the protection lightpath on $(L, I)$ and $(I, B)$ ), two links (i.e., $(L, I)$ and $(I, B)$ ) and node $I$ can also be set in sleep mode. Therefore, to minimize the overall power consumption, it is necessary to optimally route the lightpaths so that a large number of devices are supporting exclusively protection lightpaths and thus can be set in sleep mode. The problem of routing working and protection lightpaths in WDM networks in order to minimize the overall power consumption is formulated next.

\section{Network Planning at Minimum Power}

This section introduces the problem of network planning (i.e, static routing and wavelength assignment) at minimum power with devices in sleep mode (MP-S) and 1:1 dedicated path protection. To evaluate the power effectiveness of MP-S, two other planning strategies, namely planning at minimum power consumption (MP) (i.e., without sleep mode support) and planning at minimum installation cost (MC) are considered for comparison.

\section{A. Minimum Power with Devices in Sleep Mode (MP-S) Strategy}

The optimization problem for network planning at minimum power with devices in sleep mode (MP-S) is formalized as an integer linear programming (ILP) problem. The objective is to optimally route the requested working lightpaths and their 
corresponding protection lightpaths so that the power required to operate the overall network is minimized. Only the power drained by devices in active mode is considered, according to the model discussed in Section II-A. The ILP problem can be formulated as follows.

\section{Input parameters}

- $G(V, E)$ : a directed graph where $V$ is the set of vertices that represent the network nodes, $E$ is the set of arcs that represent the network links;

- $N=|V|$

- $a_{i j}$ : number of wavelengths available on link $(i, j)$;

- $C$ : large constant, e.g., $C=N \cdot \max _{(i, j)} a_{i j}$;

- $t^{s d}$ : number of lightpaths to be established from $s$ to $d$;

- $\pi^{s d, k}: k$-th pre-computed route from $s$ to $d^{2}$;

- $\phi_{n}$ : fixed power of active devices at node $n$;

- $\epsilon_{n}^{t}$ : proportional power for transmitting devices at node $n$;

- $\epsilon_{n}^{r}$ : proportional power for receiving devices at node $n$;

- $\epsilon_{n}^{s}$ : proportional power for switching devices at node $n$, such as for a path in the OXC (e.g., path in MEMS) and wavelength converters;

- $\sigma_{i j}$ : fixed power for active devices in link $(i, j)$, such as for optical amplifiers;

- $\delta_{i j}$ : proportional power for active devices in link $(i, j)$.

\section{Variables}

- integer $w^{s d, k}$ indicates the number of working lightpaths using route $\pi^{s d, k}$;

- integer $p^{s d, k}$ indicates the number of protection lightpaths using route $\pi^{s d, k}$;

- boolean $x_{n}$ indicates whether any working lightpath is passing through node $n$, i.e., determines whether all devices at node $n$ can be set in sleep mode;

- boolean $y_{i j}$ indicates whether any working lightpath is passing on link $(i, j)$, i.e., determines whether all devices on link $(i, j)$ can be set in sleep mode;

- $P_{M P-S}$ indicates the overall power consumption of the network planned using MP-S strategy.

\section{Objective function}

$$
\text { Minimize }: P_{M P-S}
$$

\section{Constraints}

$$
\begin{gathered}
P_{M P-S}=\sum_{n \in V}\left(\phi_{n} \cdot x_{n}+\epsilon_{n}^{t} \cdot \sum_{s d, k: n=s} w^{s d, k}\right. \\
\left.+\epsilon_{n}^{r} \cdot \sum_{s d, k: n=d} w^{s d, k}+\epsilon_{n}^{s} \cdot \sum_{s d, k: n \in \pi^{s d, k}, n \neq s, d} w^{s d, k}\right) \\
+\sum_{(i, j) \in E}\left(\sigma_{i j} \cdot y_{i j}+\delta_{i j} \cdot \sum_{s d, k:(i, j) \in \pi^{s d, k}} w^{s d, k}\right) \\
\sum_{k} w^{s d, k}=t^{s d} \quad \forall s, d \\
\sum_{k} p^{s d, k}=t^{s d} \quad \forall s, d
\end{gathered}
$$

\footnotetext{
${ }^{2}$ In this ILP formulation, the same set of pre-computed routes is used for working and protection lightpaths. The formulation can be easily extended to consider two different sets of pre-computed routes.
}

$$
\begin{aligned}
\sum_{k: \pi^{s d, k} \oslash \pi^{s d, j}} p^{s d, k} & \geq w^{s d, j} & & \forall s d, j \\
\sum_{s d, k:(i, j) \in \pi^{s d, k}}\left(w^{s d, k}+p^{s d, k}\right) & \leq a_{i j} & & \forall(i, j) \in E \\
\sum_{s d, k: n \in \pi^{s d, k}} w^{s d, k} & \leq x_{n} \cdot C & & \forall n \in V \\
\sum_{s d, k:(i, j) \in \pi^{s d, k}} w^{s d, k} & \leq y_{i j} \cdot C & & \forall(i, j) \in E
\end{aligned}
$$

\section{Integrality constraints}

$\begin{array}{lll}\text { integer } & w^{s d, k}, p^{s d, k} & \forall s d, k \\ \text { boolean } & x_{n} & \forall n \in V \\ \text { boolean } & y_{i j} & \forall(i, j) \in E\end{array}$

The objective (1) is the minimization of the overall network power due to fixed and proportional power consumed by devices in active mode, installed in the network links and nodes, as defined in (2). Constraints (3) and (4) select the route for the working and protection lightpaths, respectively. In constraint (5), the symbol $\oslash$ indicates the link-disjointness condition, i.e., route $\pi^{s d, k}$ and route $\pi^{s d, j}$ must be link-disjoint. Thus, the constraint forces the route of the protection lightpath to be link-disjoint with respect to the route of the working lightpath. Constraint (6) ensures that the maximum number of working and protection lightpaths passing on a link does not exceed the number of available wavelengths. Constraints (7) and (8) determine whether a node or a link, respectively, are supporting any working lightpath. Such constraints force devices supporting working lightpaths to be active. Devices not supporting any lightpath are turned off.

In the formulation, the number of variables and the number of constraints increase with of $N^{2} \cdot K$, where $K$ is the maximum number of routes between any source-destination pair. A small value of $K$ reduces the time required to solve the formulation at the expense of optimality.

\section{B. Minimum Power (MP) Strategy}

The objective of the MP problem is to minimize the overall power consumption when sleep mode is not supported by the network devices. The set of the input parameters is the same as the one used in MP-S formulation, while new variables $\bar{x}_{n}$ and $\bar{y}_{i j}$ are included and defined as follows.

\section{Variables}

- boolean $\bar{x}_{n}$ indicates whether any working or protection lightpath is passing through node $n$;

- boolean $\bar{y}_{i j}$ indicates whether any working or protection lightpath is passing on link $(i, j)$;

- $P_{M P}$ indicates the overall power consumption of the network planned using MP strategy.

\section{Objective function}

$$
\text { Minimize }: P_{M P}
$$

\section{Constraints}

Constraints (3)-(6) 


$$
\begin{aligned}
& P_{M P}=\sum_{n \in V}\left[\phi_{n} \cdot \bar{x}_{n}+\epsilon_{n}^{t} \cdot \sum_{s d, k: n=s} w^{s d, k}\right. \\
& \left.+\epsilon_{n}^{r} \cdot \sum_{s d, k: n=d} w^{s d, k}+\epsilon_{n}^{s} \cdot \sum_{s d, k: n \in \pi^{s d, k}, n \neq s, d} w^{s d, k}\right] \\
& +\sum_{(i, j) \in E}\left[\sigma_{i j} \cdot \bar{y}_{i j}+\delta_{i j} \cdot \sum_{s d, k:(i, j) \in \pi^{s d, k}}\left(w^{s d, k}+p^{s d, k}\right)\right] \\
& \sum_{s d, k: n \in \pi^{s d, k}}\left(w^{s d, k}+p^{s d, k}\right) \leq \bar{x}_{n} \cdot C \quad \forall n \in V \\
& \sum_{s d, k:(i, j) \in \pi^{s d, k}}\left(w^{s d, k}+p^{s d, k}\right) \leq \bar{y}_{i j} \cdot C \quad \forall(i, j) \in E
\end{aligned}
$$

\section{Integrality constraints}

$\begin{array}{lll}\text { integer } & w^{s d, k}, p^{s d, k} & \forall s d, k \\ \text { boolean } & \bar{x}_{n} & \forall n \in V \\ \text { boolean } & \bar{y}_{i j} & \forall(i, j) \in E\end{array}$

The objective (12) is the minimization of the overall network power due to fixed and proportional power consumed by all devices installed in the network links and nodes, as defined in (13). Constraints (14) and (15) determine whether a node or a link, respectively, are supporting any working or protection lightpath. Such constraints force devices supporting any type of lightpaths to be active. Devices not supporting any lightpath are turned off.

\section{Minimum Cost (MC) Strategy}

The objective of the MC problem is to minimize the installation cost for working and protection resources. In the following, it is assumed that the installation cost is proportional to the number of wavelengths required for the working and protection lightpaths. Among the optimal solutions for minimum cost, the solution at minimum power consumption with sleep mode support is selected. As a result, the MC formulation is based on the same input parameters and the same variables of the MP-S problem. In addition, a constant $\xi$ is introduced for tie-breaking, so that $\xi \cdot P_{M P-S}<1$.

\footnotetext{
Objective function $\quad$ Min. : $\sum_{s d, k}\left[w^{s d, k}+p^{s d, k}\right]+\xi \cdot P_{M P-S}$

Constraints Constraints (2)-(8)

Integrality constraints Constraints (9)-(11)
}

Note that MC formulation is identical to MP-S formulation, apart from the objective function whose main aim is the minimization of the resources to be installed for $P_{M P-S}$ as planning strategy.

\section{Strategy Comparsions}

The power consumption of the MP-S planning strategy is evaluated and compared against the power consumption of MP and MC strategies. The results are optimally found by running a commercially available ILP solver on the Cost 239 European network with 11 nodes and 52 unidirectional links. Each link supports 8 wavelengths, i.e., $a_{i j}=8 \forall(i, j) \in E$. Given a fixed number of requested lightpaths, a set of lightpaths is generated by uniformly selecting the source and destination nodes. The sets of lightpaths are generated until the confidence interval of the optimal network planning results are less or equal to $6 \%$ for a confidence level of $90 \%$.

A (sub)optimal solution for the proposed planning strategies is obtained considering $K=3$ shortest routes for each nodepair. The parameters characterizing the power consumption are set according to [8] as follows: $\phi_{n}=150 \mathrm{~W} \forall n \in V$ accounts for the power drained by the electronic control at each node; $\epsilon_{n}^{t}+\epsilon_{n}^{r}=5.9 \mathrm{~W} \forall n \in V$ accounts for the power drained by transmitters and receivers; $\epsilon_{n}^{s}=1.757 \mathrm{~W} \forall n \in V$ accounts for power drained by 3D MEMS per internal path through the switch (i.e., $107 \mathrm{~mW}$ ) and the optical wavelength converters (i.e., $1.65 \mathrm{~W}$ ). It assumed that each link has the same number of in-line power amplifiers and that $\sigma_{i j}=30 \mathrm{~W} \forall(i, j) \in E$ accounts for their power consumption. It is also assumed that the for an in-line power amplifier the proportional power is negligible, i.e., $\delta_{i j}=0 \forall(i, j) \in E$.

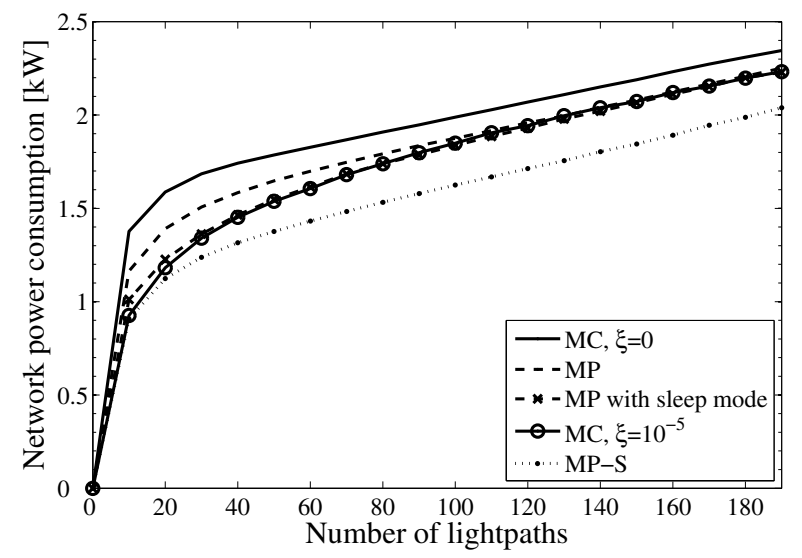

Figure 2. Network power consumption vs. number of lightpaths.

Fig. 2 shows the network power consumption as a function of the number of lightpaths to be provisioned in the network, for the three planning strategies presented in Sec. III. For benchmarking purposes, a fourth strategy (MP with sleep mode) is also presented. In this latter strategy, routing is identical to MP planning, but sleep mode is enabled for redundant devices. Results for MC planning are obtained by setting $\xi=10^{-5}$ (i.e., among the minimum cost solutions the one at minimum power is selected) and $\xi=0$ (i.e., any of the minimum cost planning solution is selected) as benchmarking.

The figure shows that minimizing the power consumption (MP planning) is effective with respect to a network planning strategy at minimum cost (MC planning with $\xi=0$ ), leading to savings of up to $10 \%$. Further reductions can be achieved by exploiting sleep mode. Indeed, exploiting sleep mode after provisioning lightpaths (i.e., MP with sleep mode) allows an additional $10 \%$ reduction of power at low loads only. If sleep mode is accounted in the planning (i.e., MP-S), savings of up to $15 \%$ at low loads and about $10 \%$ at high loads are achievable with respect to MP. This shows a) the significant savings that can be achieved by enabling sleep mode in redundant devices and b) the importance of accounting 
for sleep mode already during the network planning phase, rather than afterwards. Finally, power consumption of MC with $\xi=10^{-5}$ is higher than power consumption of MP$\mathrm{S}$, indicating that an optimal solution for installation cost may not be optimal for power consumption with sleep mode option, i.e., additional resources may be required to achieve minimum power consumption with sleep mode.

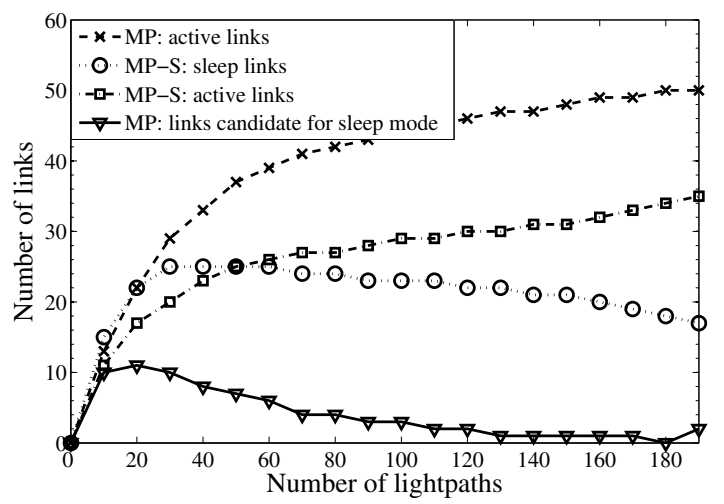

Figure 3. Total number of links in sleep and active mode vs. the number of lightpaths.

The effectiveness of a network planning strategy that accounts for sleep mode is further analyzed by the results in Fig. 3. The figure shows the number of links whose devices are set in active and sleep mode (i.e., active or sleep links respectively) for MP-S and MP. In MP-S planning, a greater number of links is set in sleep mode, while in MP planning, a large number of links is active and thus consuming more power than in MP-S. Results on the operational mode of the node devices are not reported, as only few (typically one or two) nodes are set to sleep mode in few experiments and for very low loads (i.e., 10 and 20 lightpaths), i.e., sleep mode of node devices is not exploited by the planning strategies.

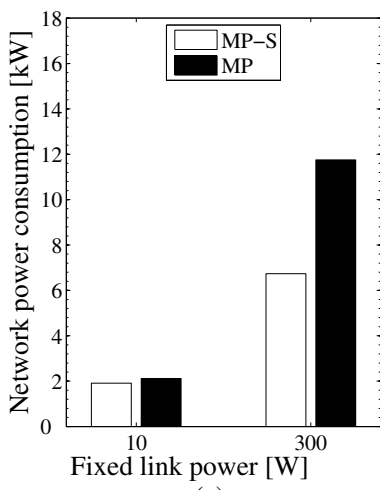

(a)

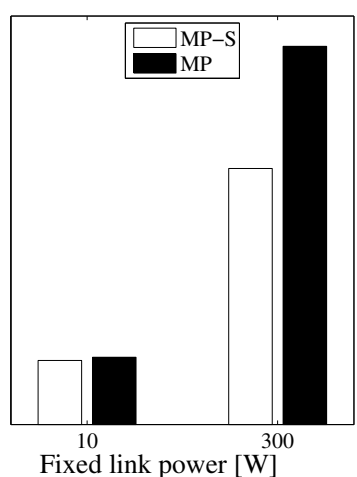

(b)
Figure 4. Network power consumption vs. fixed link power for 20 lightpaths (a) and 120 lightpaths (b).

Figs. 4 and 5 analyze the sensitivity of the solutions with the fixed link power, when the number of requested lightpaths is 20 (a) and 120 (b). Fig. 4 confirms that a planning strategy accounting for sleep mode (i.e., MP-S) is able to consistently offer better power saving when compared to MP, for any loads.

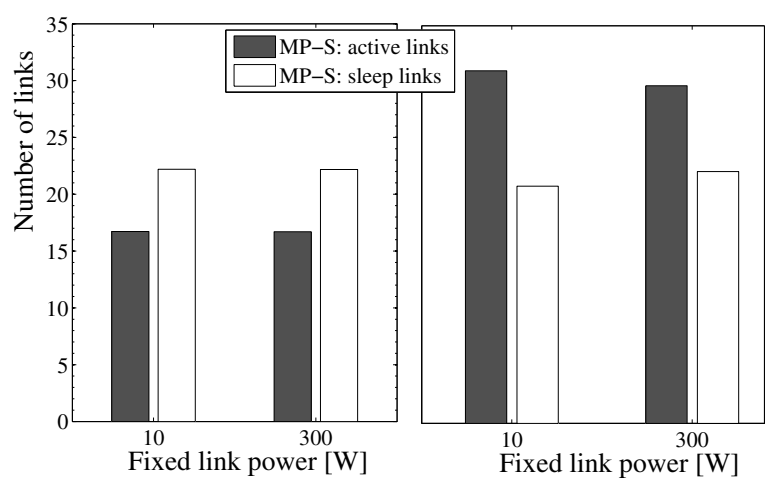

(a)

(b)

Figure 5. Total number of links in sleep and active mode vs. fixed link power for 20 lightpaths (a) and 120 lightpaths (b).

However, the power effectiveness is reduced when the fixed link power decreases. Nevertheless, MP-S planning is able to exploit the sleep mode even when the fixed link power is much lower or higher than the previously considered value (i.e., 30 W), as shown in Fig. 5. Indeed, the number of links in sleep mode is the same for the different values of fixed link power, but this may not lead to the same power savings due to the power consumption of the nodes. At low loads, the number of links in sleep mode exceeds the number of links in active mode and vice-versa at high loads (i.e., 120 lightpaths).

\section{CONCLUSION}

The paper presents an energy-efficient planning strategy for survivable WDM networks, that exploits the sleep mode of devices supporting protection lightpaths. The network planning problem is defined through an integer linear programming formulation and is solved in real case scenarios.

The optimal results show that significant power savings are achieved by properly routing the lightpaths. Link devices (such as amplifiers) supporting only protection lightpaths can be set in sleep mode, until a fault triggers a prompt re-activation. When link sleep mode is accounted during the planning, power savings can reach up to $25 \%$. It was also found that sleep mode at nodes can be exploited, but in very specific conditions, e.g., at low loads. The achieved benefits are calling for support of sleep mode in optical devices, as advocated by [7].

\section{REFERENCES}

[1] The Climate Group, "SMART 2020: Enabling the low carbon economy in the information age," Global eSustainability Initiative, Tech. Rep., 2008.

[2] I. Cerutti, L. Valcarenghi, and P. Castoldi, "Power saving architectures for unidirectional WDM rings," in Proc. OFC, march 2009, pp. 1 -3.

[3] E. Yetginer and G. N. Rouskas, "Power efficient traffic grooming in optical WDM networks," in Proc. Globecom, December 2009.

[4] Y. Wu, L. Chiaraviglio, M. Mellia, and F. Neri, "Power-aware routing and wavelength assignment in optical networks," in Proc. ECOC, 2009.

[5] G. Shen and R. Tucker, "Energy-minimized design for ip over wdm networks," IEEE/OSA JOCN, vol. 1, no. 1, pp. 176-186, June 2009.

[6] C. Ou et al., Survivable Optical WDM Networks, Springer, 2005.

[7] Energy Star, "Small network equipment," http://www.energystar.gov/index.cfm?c=new_specs.small_network_equip.

[8] S. Aleksic, "Analysis of power consumption in future high-capacity network nodes," IEEE/OSA JOCN, vol. 1, no. 3, 2009. 